\title{
PHARMASAT: DRUG DOSE DEPENDENCE RESULTS FROM AN AUTONOMOUS MICROSYSTEM-BASED SMALL SATELLITE IN LOW EARTH ORBIT
}

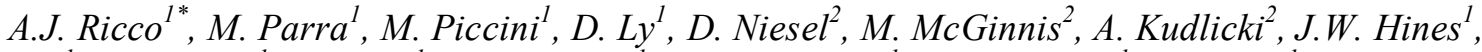

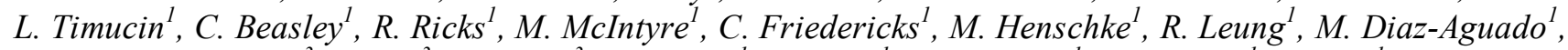

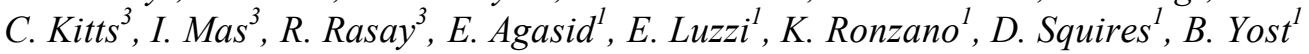 \\ ${ }^{1}$ Small Spacecraft Division, NASA Ames Research Center, Moffett Field, California, USA \\ ${ }^{2}$ University of Texas Medical Branch, Galveston, Texas, USA \\ ${ }^{3}$ Santa Clara University, Santa Clara, California, USA
}

\begin{abstract}
We designed, built, tested, space-qualified, launched, and downlinked bioanalytical data from PharmaSat, the first fully autonomous outer-space pharmaceutical dose-response bioanalytical system on a free-flying satellite. PharmaSat tracks microorganism culture population density and metabolic activity in 48 microwells via 3-color optical absorbance. Its $5.1-\mathrm{kg}$ total mass includes solar cells, integrated spacecraft "bus" module (power/batteries/ control/communications), and a sealed containment vessel housing the biofluidic, optical, thermal, and sensor subsystems. Data were obtained from all subsystems over several days following nutrient introduction to initiate growth of Saccharomyces cerevisiae, followed by challenges with three dose levels of an antifungal agent.
\end{abstract}

\section{INTRODUCTION}

Long-term spaceflight affects living organisms. Data for mammals are consistent with immune stress, decreased bone density, muscle atrophy, and slowed wound healing. For cells and microorganisms in culture, absence of gravity means thermal gradients do not cause convection as on Earth, potentially altering mass transport that influences nutrient delivery and waste removal. For all living organisms, rates of damage from high-energy radiation increase measurably outside the shielding of Earth's magnetosphere. In microbes, rates and extent of growth vary, and pathogen virulence has been reported to change [1]. Astronauts can contract infections; effective treatment may involve changes in therapy
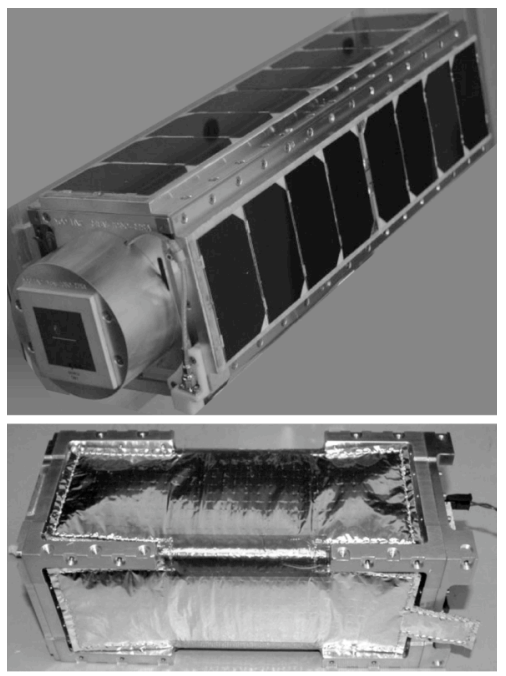

Figure 1: Integrated PharmaSat freeflying satellite $(\sim 35 \mathrm{~cm}$ long, $5.1 \mathrm{~kg})$ with solar panels, patch antenna on near end (top); payload frame and insulation-wrapped pressure vessel (bottom) containing the integrated culturing and bioanalytical system. cost and other limitations of human-tended space biological experiments while expanding the range of launch opportunities.

On May 19, 2009, the 5.1-kg PharmaSat spacecraft launched as a secondary payload aboard a Minotaur I rocket from Wallops Flight Facility, Virginia. This "hitchhiker" approach to space science offers much lower launch costs (about $\$ 40,000 / \mathrm{kg}$ ) than dedicated space biology missions, but can add operational constraints: PharmaSat was delivered for test and launch-vehicle integration nearly 7 weeks before launch; no power was available for thermal control prior to deployment in space, although temperature was maintained between 4 and $37^{\circ} \mathrm{C}$. As an add-on payload, PharmaSat was mounted, using a vibration-damping fastener, to the Minotaur I upper-stage rocket motor; calculations predicted $<5{ }^{\circ} \mathrm{C}$ temperature increase inside the pressure vessel containing the biofluidic subsystem during the short burn time of this motor.

About 20 min after launch, PharmaSat was spring-ejected into low-Earth orbit (460 km elevation) as a free-flying satellite. It functioned nominally; within days, results from the PharmaSat microgravity 48-microwell drug-dose-dependence experiment were telemetered to Earth (http://www.nasa.gov/mission_pages/smallsats /pharmasat/main/index.html). Leveraging multiple micro and miniature technologies, PharmaSat is the first fully autonomous outer-space pharmaceutical dose-response analytical system. Here we report its design, development, and the spaceflight and groundcontrol experimental results from this integrated bioanalytical system.

\section{DESIGN AND CONSTRUCTION}

PharmaSat's 5.1 $\mathrm{kg}$ mass comprises a structure approximately $10 \times 10 \times 35 \mathrm{~cm}$, Figure 1 , including triplejunction solar cells; patch antenna for 2.4$\mathrm{GHz}$ communication; mechanical structure; spacecraft power, processor, communications, and control system (collectively, the "bus"); and the experimental containment vessel, internal
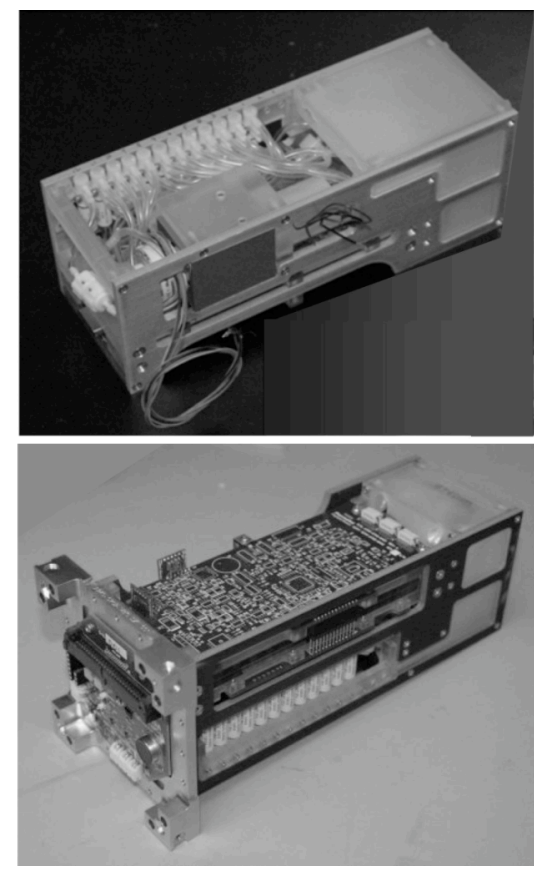

Figure 2: Two views of PharmaSat integrated payload (which slides inside the pressure vessel shown in Figure 1). It includes fluidic, optical, thermal, sensor, and electronics subsystems. 


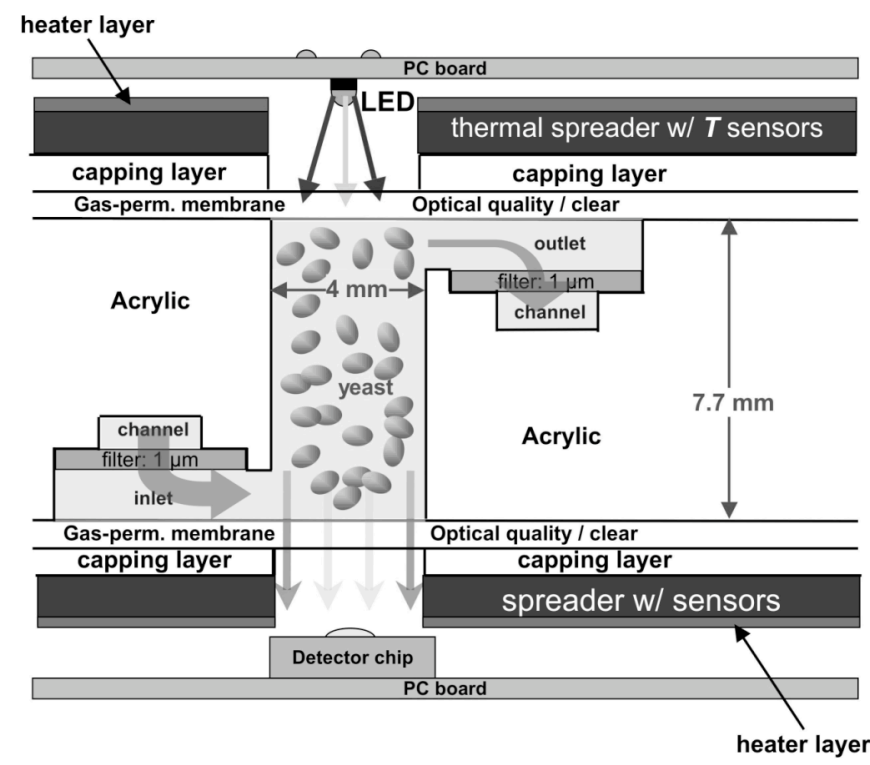

Figure 3: Microfluidic, optical, and thermal cross section of one of 48 wells; each contains $100 \mu L$ and has integral 1.2- $\mu m$ filter membranes at inlet and outlet to confine the yeast. RGB LED and detector pair at opposite ends of each well measure 3-color transmittance. Patterned Kapton heaters plus aluminum thermal spreaders give $<0.3{ }^{\circ} \mathrm{C}$ temperature uniformity across the card.

volume 1.2 L (Figures 1, 2), which houses the bio/fluidic, optical, thermal, sensor, and payload electronics subsystems (Figure 2). Magnetic rods passively orient the satellite, aiming its long axis and hence antenna toward Earth's N magnetic pole when the spacecraft is above the northern hemisphere. Magnetic hysteresis rods damp "wobble" in spacecraft motion by dissipating energy when they move in Earth's magnetic field.

\section{Fluidic SubSystem}

The PharmaSat fluidic card (cross section: Figure 3) includes forty-eight $100-\mu \mathrm{L}$ culture wells, $4 \mathrm{~mm}$ diameter $\times 7.8 \mathrm{~mm}$ deep, and 11 solid-state reference wells. 9-mm pitch along rows and 9and 13.5-mm spacing between rows allow card optical analysis with standard multiwell plate readers. The card was fabricated by Micronics, Inc. from laser-cut poly(methylmethacrylate) layers (Figure 4) laminated with pressure-sensitive adhesive interlayers supported on (poly(ethylene terephthalate)) carrier films. Four independent sets of manifolded fluidic inlets and outlets with 1.2$\mu \mathrm{m}$ nylon fiber membrane filters (Sterlitech) supply nutrients and antifungal agent to the four 12-well banks containing the yeast.

Covering tops and bottoms of the fluidic cards is a $51-\mu \mathrm{m}-$ thick layer of optical-quality poly(styrene) (PS), which provides gas permeability for $\mathrm{CO}_{2}$ and $\mathrm{O}_{2}$ exchange. Cards were fabricated without a top cover layer, sterilized using ethylene oxide, offgassed in vacuum for 2 weeks at $55^{\circ} \mathrm{C}$, and stored in sterile bags.

Inoculation of biowells with microorganisms and fluid filling of fluid storage bags, tubing, channels, and wells are carried out under sterile conditions prior to payload integration. To prepare a card for laboratory or flight experiments, solid-state colored optical reference elements are placed in the eleven "dry" wells. Each biowell is inoculated with $10 \mu \mathrm{L}$ of dormant $S$. cerevisiae culture, to provide a final density of $5-10 \times 10^{5} / \mathrm{mL}$, with care to avoid wetting filter membranes. To help eliminate gas bubbles, wells and channels are purged with $\mathrm{CO}_{2}$. The open wells are then covered by a second PS film. Channels and inoculated biowells are next filled with thoroughly vacuum-degassed sterile water; any

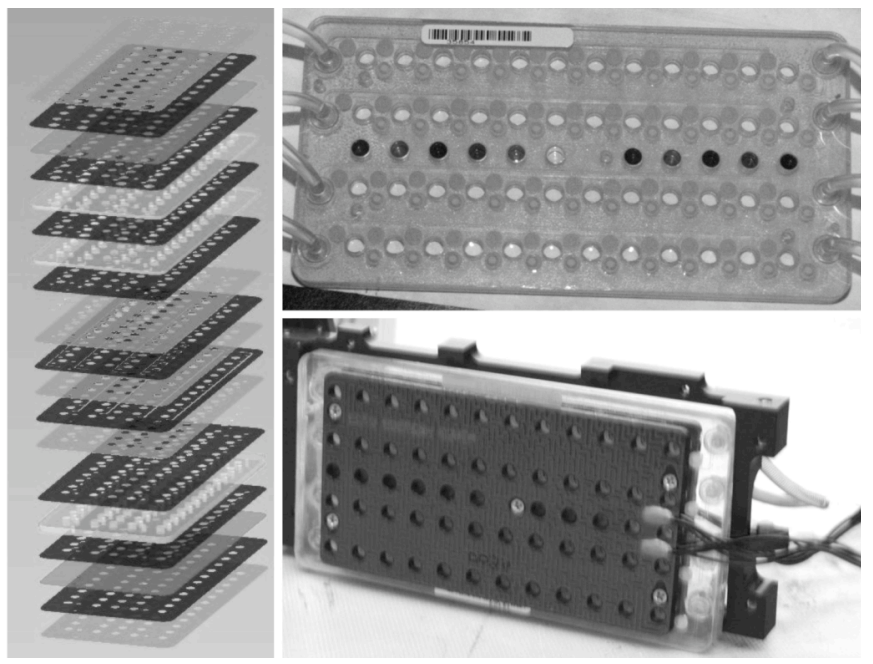

Figure 4: Exploded view of fluidic card showing laser-cut acrylic and PSA layer stack (left). Completed card (upper right) shows 4 banks of 12 wells each and 11 reference wells (colored, along center line). A pair of patterned Kapton heater films with aluminum spreader plates (bottom) sandwiches the fluidic card.

remaining $\mathrm{CO}_{2}$ bubbles dissolve gradually as stasis medium flows through the wells and channels.

The fluid-delivery-and-dosing system, Figure 5, comprises 14 solenoid-operated valves (The Lee Co.); a piston-type metering pump (Lee) and a diaphragm-based pump (KNF Neuberger); a bubble trap (developed in-house); nine medical-grade fluoropolymer bags (American Fluoroseal) for growth medium, viability dye, antifungal agent, and waste; and medical-grade connecting tubing (Sanipure 60 tubing, Saint-Gobain Performance Plastics).

The fluidic system supports organism dormancy, supplies growth medium to initiate organism growth in orbit, and mixes, meters, and supplies the antifungal agent, voriconazole, at three concentrations. The fluidics card and system maintain the microwells in a bubble-free state for 5 - 7 weeks after loading and assembly, despite the permeability of the covering membranes to water vapor. This issue was mitigated by filling the waste bag for

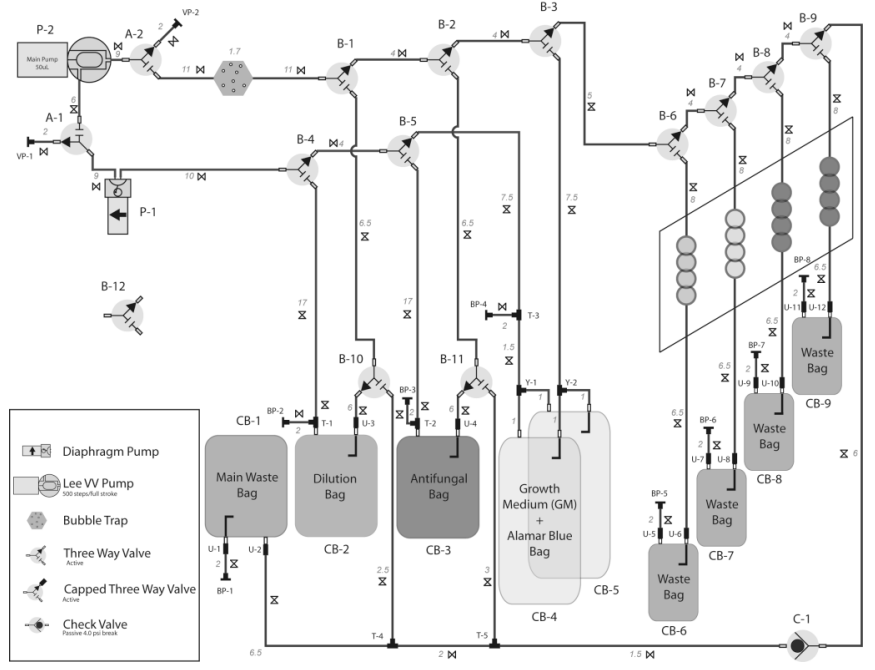

Figure 5: Block diagram of fluid delivery and dosing system. The variable-volume $(V V)$ metering pump makes precise dilutions of the antifungal agent for delivery at 3 concentrations. The diaphragm pump provides higher flow rates for circulation and mixing. Each bank of 12 wells has a dedicated waste bag, while the growth medium, antifungal agent, dilution, and mixing bags are common. 
each well bank with several $\mathrm{mL}$ of stasis buffer maintained at a low "back pressure", replacing water lost to evaporation and preventing bubble formation. Evaporation was also mitigated by the sealed pressure vessel, the internal air volume $(<0.4 \mathrm{~L})$ of which was humidified to near saturation by evaporation from the fluidic card.

\section{Optical, Thermal, and Sensor SubSystems}

Each of the 48 fluidic and 11 solid-state reference wells has a dedicated optical sub-assembly (Figure 3), comprised of a 3-color LED (Lite-On) providing illumination bands at 470, 525, and 615 $\mathrm{nm}$ with 26,35 , and $18 \mathrm{~nm}$ spectral half-widths, respectively, and an intensity-to-frequency ASIC (Texas Advanced Optoelectronic Solutions) providing $>5$ decades of digital output. Only a single LED wavelength band at a single well is energized at once, eliminating any possibility of optical crosstalk.

The optical system measures organism growth in two ways: optical density changes due to light scattering by the yeast cells, which is directly proportional to cell number [6], and color change of a "viability" dye, alamar blue (AB), which is deliberately added to the growth medium. $\mathrm{AB}$ changes from blue (oxidized form) to pink (reduced form) when enzymes generated by metabolic processes in cells act upon it [7]. AB's blue form has its absorbance maximum at $600 \mathrm{~nm}$, the pink at $570 \mathrm{~nm}$. To first order, the red LED tracks the concentration of oxidized $A B$, the green is sensitive to the pink form, and the blue, where neither pink nor blue forms of the dye absorbs particularly strongly, responds mainly to light scattering by the yeast cells.

The match of the three LED bands to the three optical parameters of interest is, unfortunately, imperfect. We measured optical absorbance "cross terms", finding the three most important of them to be (1) absorption of the blue band by the blue form of $\mathrm{AB}(10 \%$ of its absorbance at $615 \mathrm{~nm}$ ); (2) absorption of the blue band by the pink form of $\mathrm{AB}$ ( $29 \%$ of its absorbance at $525 \mathrm{~nm}$ ); (3) absorption

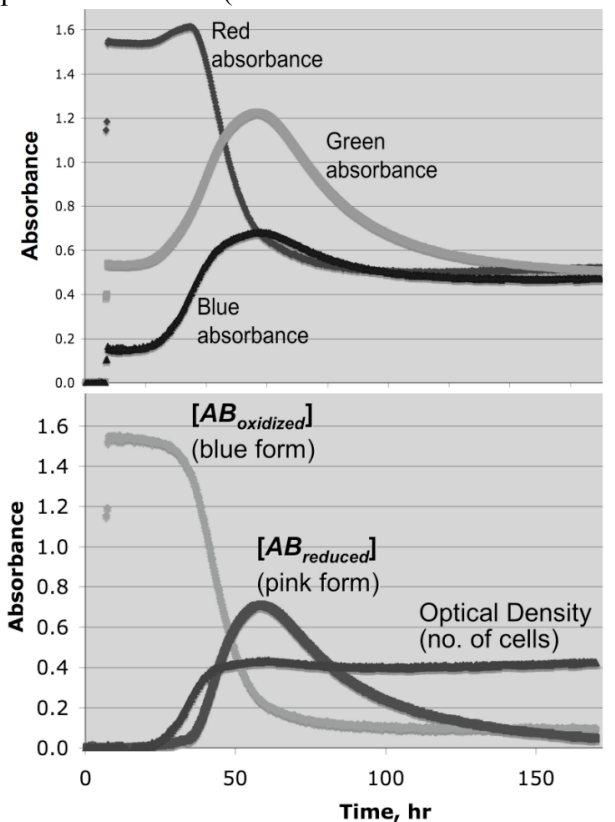

Figure 6: Three-color absorbance of one microwell of growing yeast measured with the PharmaSat optical system. Top: As-measured absorbances, corrected for known absorbance of initial concentration of blue form of alamar blue. Bottom: Absorbances after correction for "cross terms", resulting in absorbances of the two forms of $A B$ and the optical density due to light scattering by the yeast cells.

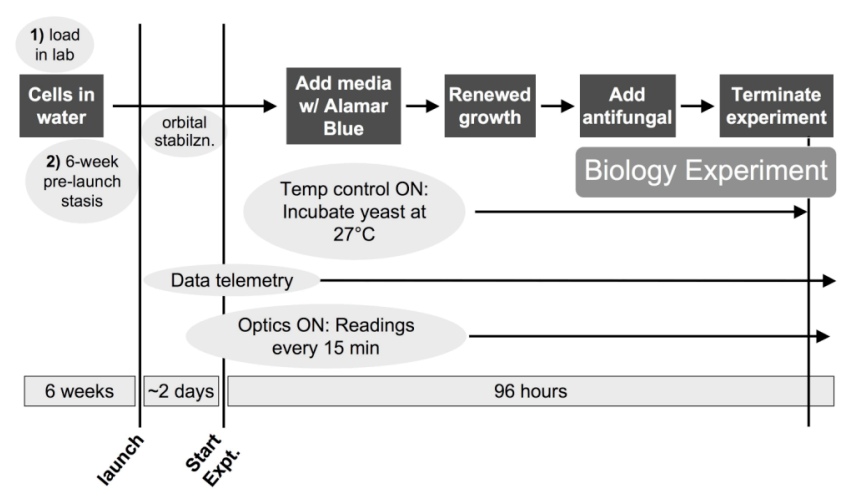

Figure 7: Operational timeline for PharmaSat space experiment.

mizing light reflections) as thermal spreaders sandwiching the fluidic card (Figure 3). A "bang-bang" controller operated by the system microcontroller provides temperature stability of $<0.3{ }^{\circ} \mathrm{C}$. Average power consumption by the thermal systems was $\sim 2 \mathrm{~W}$ to maintain the card at the $27^{\circ} \mathrm{C}$ growth temperature while in orbit.

Sensors in the pressure vessel include six for temperature (Analog Devices) distributed over the thermal spreaders to monitor fluidic card temperature and provide the control signal; a micromachined pressure transducer (Motorola); and a thin-film capacitive humidity sensor (Sensirion). In the spacecraft bus, acceleration is monitored in 3 axes by MEMS accelerometers (Silicon Designs); a PIN diode (Hamamatsu) monitors radiation flux.

\section{RESULTS FROM ORBIT AND GROUND CONTROLS}

Figure 7 shows the operational timeline for the PharmaSat space experiment. Nearly 7 weeks after integration with the rocket, PharmaSat launched on May 19, 2009; it was deployed $20 \mathrm{~min}$ later. Radio contact was established within hours and its orbit passively stabilized. Orbital trajectory puts PharmaSat in sunlight $~$ $2 / 3$ and darkness $\sim 1 / 3$ of each 97 -min orbit. Li-ion batteries in the bus are charged by the solar panels, providing uninterrupted power.

Following stabilization (gravity $<10^{-3} \mathrm{xg}$, calculated from variations in currents from the 4 solar panels), some $47 \mathrm{hr}$ after orbital deployment, PharmaSat changed the temperature of the fluidic card from ambient $\left(\sim 6-16^{\circ} \mathrm{C}\right.$, depending on the phase of orbit) to $27{ }^{\circ} \mathrm{C}$. With temperature stabilized, S. cerevisiae (strain: BY 4743) growth was initiated $3.6 \mathrm{hr}$ later in all 48 wells by pumping in a $3 x$ exchange of RPMI-1640 nutrient medium (as in [8], omitting phenol red) containing alamar blue viability dye $(10 \%$ of stock), one 12-well bank at a time, displacing the stasis buffer.

Meaningful measurements of AF dose dependence require the antifungal agent to be introduced when the yeast are growing stably but prior to the culture reaching high density, when the AF generally becomes ineffective. Because growth rate in the microgravity environment was not known in advance, growth progress was monitored by telemetry; based on initial growth rates, AF was administered approximately $12 \mathrm{hr}$ after initial RPMI introduction.

Using an identical satellite system in the ground laboratory, housed in a thermal chamber to mimic temperatures reported from the satellite, and with $S$. cerevisiae loaded from the same prepared cultures within two days of the flight loading of yeast, a "delayed synchronous" ground control experiment was conducted in order to meaningfully compare ground and space results.

Figure 8 compares the results from the ground-control experiment (top) with the spaceflight results, telemetered from orbit (bottom). The data shown here are corrected absorbances of alamar blue throughout the growth and challenge process in its oxidized form, as measured using the red LED and after correction for "cross terms" described above. After initial growth, a growth medium/AF 


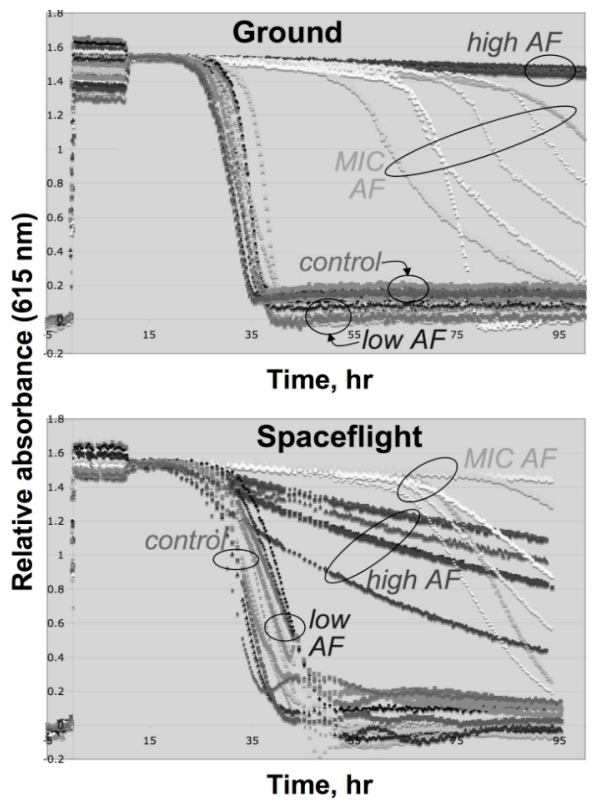

Figure 8: Terrestrial (top) and spaceflight (bottom) growth curves for $\mathrm{S}$. cerevisiae at 27 ${ }^{\circ} \mathrm{C}$ in RPMI growth medium as a function of voriconazole antifungal concentration. 6 wells are tracked for each condition: "Control" includes no AF; "low AF", "MIC", and "high AF" correspond to 0.13, 0.50, and 2.0 $\mu \mathrm{g} / \mathrm{mL}$ concentrations of voriconazole, respectively. (Circles and ellipses indicate sets of growth curves for each labeled condition).

$=6$ independent microwells, which were initially loaded with yeast corresponding to an approximate optical density (OD) of 0.1 .

Because the delay between loading the organisms and deployment in space could not be predicted exactly (weather and other factors can, and do, cause launch delays), the remaining 6 wells of each bank of 12 were loaded with yeast at an OD of 0.2: in the event that insufficient organisms survived the stasis period in the $\mathrm{OD}=0.1$ groups of wells, the $\mathrm{OD}=0.2$ wells were included to provide an extra $100 \%$ population margin for initiating growth. Because AF dose dependence tests are typically more reliable at lower yeast population densities, the successful and stable growth of all 24 wells containing the OD $=0.1$ cultures led us to choose those data for our initial ground/flight comparisons.

Figure 9 analyzes $\mathrm{AF}=0$ yeast growth rates by two measures, both of which indicate slower growth $(p<0.04)$ in space's microgravity environment than on Earth. In a significant gravitational field, even small thermal gradients, as might, for example, be caused by the on/off cycling of the fluidic card heater, can result in thermal convection in an otherwise quiescent culture (due to fluid density differences). In contrast, in microgravity conditions, fluid density differences do not drive convection. Thus, it may be the case that the yeast grow more slowly in space because the transport of nutrients to the cells and/or the transport of waste products away from the cells is slower, being driven solely by diffusion, than on Earth where convection can play a role in mass transport.

Figure 8 shows that the "low AF" condition, $\mathrm{AF}=0.13$ $\mu \mathrm{g} / \mathrm{mL}$, does not significantly affect growth parameters relative to zero AF. Thus, spaceflight growth is slower by about the same relative factor at this low AF concentration as in the absence of AF.

At the MIC, both ground and spaceflight data show significant spread in the 9 growth curves for $\mathrm{AF}=0.5 \mu \mathrm{g} / \mathrm{mL}$. This was ex- pected, as the growth rates with the antifungal near its MIC are very sensitive to small differences in concentration, temperature, and other parameters. Interestingly, the spaceflight data show roughly the same slowing of growth at the MIC as they do at the zero and low AF concentrations.

At high antifungal concentrations $(\mathrm{AF}=2.0$ $\mu \mathrm{g} / \mathrm{mL})$, an interesting difference is observed: as expected, terrestrial data show near-total inhibition of growth. In space, however, Figure 8 reveals significant metabolic activity, as measured by the decrease in $[\mathrm{AB}]$ with time for all 6 wells. We speculate that diffusiononly mass transport may allow yeast to continue limited metabolic activity at higher $\mathrm{AF}$ concentrations in space than on Earth. If verified (ultimately in animal models),
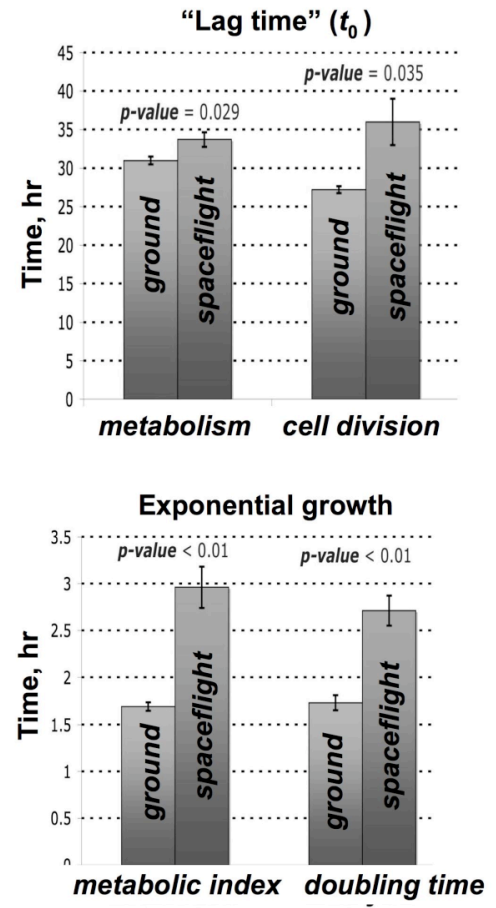

Figure 9: Comparison of "lag time" before yeast growth begins (top) and the time constant of the exponential growth phase (bottom) for ground and spaceflight measurements with zero antifungal agent concentration.

this could have significant implications for the treatment of fungal infections in the space environment.

\section{REFERENCES}

[1] J.W. Wilson, C.M. Ott, ..., and C.A. Nickerson, "Space Flight Alters Bacterial Gene Expression and Virulence and Reveals a Role for Global Regulator Hfq," PNAS, 104, 16299 (2007).

[2] T.G. Hammond, F.C. Lewis, T.J. Goodwin, et al., "Gene Expression in Space," Nature Medicine, 5, 359 (1999).

[3] N.F. deRooij, S. Gautsch, et al., "MEMS for Space", Proc. Transducers 2009, IEEE: New York (2009); pp. 17-24.

[4] J.J. Cefai, D.A. Barrow, P. Woias, and E. Muller, "Integrated Chemical Analysis Microsystems in Space Life Sciences Research," J. Micromech. Microeng., 4, 172 (1994).

[5] A.J. Ricco, J.W. Hines, et al., "Autonomous Genetic Analysis System to Study Space Effects on Microorganisms: Results," Proc. Transducers 2007, IEEE: New York (2007); pp. 33-37.

[6] A.J. Ricco, E. Agasid, et al., "Integrated System to Analyze the Genetic Effects of the Space Environment on Living Cells in Culture: GeneSat," Proceedings $\mu$ TAS 2005, Transducers Research Foundation: Cleveland (2005), pp. 527-529.

[7] J. O'Brien, I. Wilson, et al., "Investigation of the Alamar Blue (Resazurin) Fluorescent Dye for the Assessment of Mammalian Cell Cytotoxicity, Eur. J. Biochem., 267, 5421 (2000).

[8] "Reference Method for Broth Dilution Antifungal Susceptibility Testing of Yeasts. Approved Standard M27-A2", 2nd ed. National Committee for Clinical Laboratory Standards, Villanova, PA (2002); Appendix A and Table 6.

\section{CONTACT}

*A.J. Ricco, tel: +1-650-604-4276; antonio.j.ricco@nasa.gov 